\title{
Isotropic steady states in galactic dynamics revised
}

\author{
Yan Guo \\ Lefschetz Center for Dynamical Systems \\ Division of Applied Mathematics \\ Brown University, Providence, RI 02912 \\ and \\ Gerhard Rein \\ Mathematisches Institut der Universität München \\ Theresienstr. 39 \\ 80333 München, Germany
}

\begin{abstract}
The present paper completes our earlier results on nonlinear stability of stationary solutions of the Vlasov-Poisson system in the stellar dynamics case. By minimizing the energy under a mass-Casimir constraint we construct a large class of isotropic, spherically symmetric steady states and prove their nonlinear stability against general, i. e., not necessarily symmetric perturbations. The class is optimal in a certain sense, in particular, it includes all polytropes of finite mass with decreasing dependence on the particle energy. 1
\end{abstract}

\footnotetext{
${ }^{1}$ This work was published as GuO, Y., ReIN, G.: Isotropic steady states in galactic dynamics. Commun. Math. Phys. 219, 607-629 (2001). We correct an error in the analysis of the limiting "Plummer case" which was pointed out to us by Y.-H. Wan: In the proof of the former Lemma 7 we used the "identity" $\int f^{*}(x, v) d v=\left(\int f(x, v) d v\right)^{*}$ where $*$ denotes the symmetric decreasing rearrangement with respect to $x$. This is clearly false, and we modify Section 6 accordingly and also a detail in the proof of Theorem 3.
} 


\section{Introduction}

The question of which galaxies or globular clusters are stable has for many years attracted considerable attention in the astrophysics literature, cf. [4, 6] and the references there. If one neglects relativistic effects and collisions among the stars, then from a mathematics point of view the question is which steady states of the Vlasov-Poisson system

$$
\begin{gathered}
\partial_{t} f+v \cdot \partial_{x} f-\partial_{x} U \cdot \partial_{v} f=0, \\
\triangle U=4 \pi \rho, \lim _{|x| \rightarrow \infty} U(t, x)=0, \\
\rho(t, x)=\int f(t, x, v) d v,
\end{gathered}
$$

are stable. Here $f=f(t, x, v) \geq 0$ denotes the density of the stars in phase space, $t \in \mathbb{R}$ denotes time, $x, v \in \mathbb{R}^{3}$ denote position and velocity respectively, $\rho$ is the spatial mass density of the stars, and $U$ the gravitational potential which the ensemble induces collectively.

If $U_{0}$ is a time-independent potential then the particle energy

$$
E=\frac{1}{2}|v|^{2}+U_{0}(x)
$$

is conserved along the characteristics of the Vlasov equation. Therefore, a standard technique to obtain steady states of the Vlasov-Poisson system is to prescribe the particle distribution $f_{0}$ as a function of the particle energythis takes care of the Vlasov equation-and to solve self-consistently the remaining Poisson equation. The main problem then is to show that the resulting steady state has finite mass and possibly compact support. A well known class of steady states for which this approach works are the so-called polytropes

$$
f_{0}(x, v)=\left(E_{0}-E\right)_{+}^{k} .
$$

Here $(\cdot)_{+}$denotes the positive part, $E_{0} \in \mathbb{R}$ is a cut-off energy, and $-1 / 2<$ $k \leq 7 / 2$; only for this range of exponents do these steady states have finite mass, if $k<7 / 2$ they have compact support in addition. If $f_{0}$ depends only on the particle energy the resulting steady state is isotropic and spherically symmetric. Assuming spherical symmetry of $U_{0}$ to begin with steady states 
may also depend on a further conserved quantity, the modulus of angular momentum squared,

$$
L:=|x|^{2}|v|^{2}-(x \cdot v)^{2},
$$

in which case they are no longer isotropic. According to Jeans' Theorem the distribution function of any spherically symmetric steady state has to be a function of the invariants $E$ and $L$, cf. [2].

In [7, 9, 10, 17] we addressed the stability of steady states by a variational technique: It was shown that an appropriately chosen energy-Casimir functional has a minimizer under the constraint that the mass is prescribed, this minimizer was shown to be a steady state, and its nonlinear stability was derived from its minimizing property. While this turned out to be an efficient method to assess the stability of known steady states and also to construct new ones which automatically have finite mass, compact support, and are stable, there were two unwanted restrictions: Perturbations had to be spherically symmetric, and only the polytropes with $0<k<3 / 2$ were covered. A physically realistic perturbation, say by the gravitational pull of some distant galaxy, is hardly spherically symmetric. Also, while the restriction $k>0$ was indispensable - for $k \leq 0$ no corresponding Casimir functional can be defined - and is probably necessary for stability since it makes $f_{0}$ a decreasing function of the particle energy, the restriction $k<3 / 2$ is less well motivated. In [8] the first author removed the latter restriction in the case of the polytropes, while in [18 the second author removed the restriction to spherically symmetric perturbations for a class of isotropic steady states including the polytropes with $k<3 / 2$. It is the purpose of the present paper to combine these techniques to obtain a result which we believe is optimal in the following sense: It does not require any symmetry restrictions of the perturbations, and it covers all isotropic polytropes. As a matter of fact, the restrictions we require of the steady states are necessary to guarantee finite mass and to make the distribution function a decreasing function of the energy. Presumably, if the latter condition is violated sufficiently strongly, then the steady state is unstable.

The new elements in our analysis which allow for the improvements described above are the following: Previously we minimized an energy-Casimir functional under a mass constraint. Now we minimize the total energy of the system under a mass-Casimir constraint. The change of the role of the Casimir functional-from part of the minimized functional into part of the 
constraint - allows us to remove the restriction $k<3 / 2$ and was introduced in [8]. It also leads to a much cleaner assumption on the steady state or on the Casimir functional respectively. Inspired by the concentration-compactness argument due to P. L. Lions [14, which was used in [18, we use a refinement of our previous scaling and splitting argument in the compactness analysis of the energy functional to get rid of the symmetry assumption for the perturbations.

The investigation is restricted to isotropic steady states: If one includes anisotropic ones then the Casimir functional is not conserved along not spherically symmetric solutions, and the method breaks down. Anisotropic steady states are - under the appropriate assumptions - stable against spherically symmetric perturbations. Whether or not they are stable against general perturbations remains an open problem.

The paper proceeds as follows: In the next section we establish some preliminary estimates which in particular show that the total energy is bounded from below and the kinetic energy is bounded along minimizing sequences. In Section 3 the existence of a minimizer of the energy is established. To prevent mass from running off to spatial infinity along a minimizing sequence we analyze how the total energy behaves under scaling transformations and under splittings of the distribution into different pieces. In Section 4 we show that such minimizers are spherically symmetric steady states of the Vlasov-Poisson system with finite mass and compact support. The stability properties of the steady states are then discussed in the Section 5. Here we point out one problem: If $f_{0}$ is a steady state then $f_{0}(x+t V, v+V)$ for any given velocity $V \in \mathbb{R}^{3}$ is a solution of the Vlasov-Poisson system which for $V$ small starts close to $f_{0}$, but travels away from $f_{0}$ at a linear rate in $t$. This trivial "instability", which cannot be present for spherically symmetric perturbations, is handled by comparing $f_{0}$ with an appropriate shift in $x$-space of the time dependent perturbed solution $f(t)$. Technically, the necessity of this shift arises in the application of our compactness argument. The considerations discussed so far are restricted to Casimir functionals satisfying a growth condition which excludes the polytropic case $k=7 / 2$. This limiting case, the so-called Plummer sphere, is investigated in the final section. It poses additional difficulties due to a particular scaling invariance of the various functionals considered, but by using rearrangements we are able to reduce it to the same problem with symmetry, which has been investigated in [8]. This shows that is can be essential to understand the symmetric case 
first, and we hope that such a reduction to symmetry can be applied to other problems as well.

We conclude the introduction with some references, where we also compare our approach with other approaches to the stability problem. The first nonlinear stability result for the Vlasov-Poisson system in the present stellar dynamics case is due to G. WolAnsky 22]. It is restricted to spherically symmetric perturbations of the polytropes

$$
f_{0}(x, v)=\left(E_{0}-E\right)_{+}^{k} L^{l}
$$

with exponents $l>-1,0<k<l+3 / 2$ with $k \neq-l-1 / 2$ and uses a variational approach for a reduced functional which is not defined on a set of phase space densities $f$ but on a set of mass functions $M(r):=\int_{|y| \leq r} \rho(y) d y$ with $r \geq 0$ denoting the radial coordinate. In particular, is does not yield a stability estimate directly for the phase space distribution $f$. In 21] Y.-H. WAN proves stability by a careful investigation of the quadratic and higher order terms in a Taylor expansion of the energy-Casimir functional about a steady state. He has to assume the existence of the steady state, requires a strong condition on $f_{0}$ which is satisfied by the polytropes only for $k=1$ and $l=$ 0 , but his arguments do not require spherical symmetry of the admissible perturbations. We also mention [四] where stability for the limiting polytropic case $k=7 / 2$ and $l=0$ is considered. Global classical solutions to the initial value problem for the Vlasov-Poisson system were first established in [15], cf. also [20]. A rigorous result on linearized stability is given in [3]. For the plasma physics case, where the sign in the Poisson equation is reversed, the stability problem is better understood; we refer to [0, 11, 12, 16]. Finally, a very general condition which guarantees finite mass and compact support of steady states, but not their stability, is established in [19.

\section{Preliminaries}

For a measurable function $f=f(x, v)$ we define

$$
\rho_{f}(x):=\int f(x, v) d v, x \in \mathbb{R}^{3},
$$

and

$$
U_{f}:=-\rho_{f} * \frac{1}{|\cdot|} .
$$


Next we define

$$
\begin{aligned}
E_{\text {kin }}(f) & :=\frac{1}{2} \iint|v|^{2} f(x, v) d v d x \\
E_{\text {pot }}(f) & :=-\frac{1}{8 \pi} \int\left|\nabla U_{f}(x)\right|^{2} d x=-\frac{1}{2} \iint \frac{\rho_{f}(x) \rho_{f}(y)}{|x-y|} d x d y, \\
\mathcal{H}(f) & :=E_{\text {kin }}(f)+E_{\text {pot }}(f),
\end{aligned}
$$

and

$$
\mathcal{C}(f):=\iint Q(f(x, v)) d v d x
$$

where $Q$ is a given function satisfying certain assumptions specified below. We will minimize the total energy or Hamiltonian $\mathcal{H}$ of the system under a mass-Casimir constraint, i. e., over the set

$$
\mathcal{F}_{M}:=\left\{f \in L^{1}\left(\mathbb{R}^{6}\right) \mid f \geq 0, \mathcal{C}(f)=M, E_{\text {kin }}(f)<\infty\right\},
$$

where $M>0$ is prescribed. The function $Q$ has to satisfy the following

Assumptions on $Q: Q \in C^{1}([0, \infty[), Q \geq 0, Q(0)=0$, and

(Q1) $Q(f) \geq C\left(f+f^{1+1 / k}\right), f \geq 0$, with constants $C>0$ and $0<k<7 / 2$,

(Q2) $Q$ is convex.

\section{Remark.}

(a) In the last section we consider the limiting case $k=7 / 2$ for which

$$
Q(f):=f^{9 / 7}, f \geq 0 .
$$

(b) On their support the minimizers obtained later will satisfy the relation

$$
\lambda_{0} Q^{\prime}\left(f_{0}\right)=E
$$

with some Lagrange multiplier $\lambda_{0}<0$ and $E$ as defined in (1.1). Thus $f_{0}$ is a function of the particle energy and thus a steady state of the Vlasov-Poisson system, provided this identity can be inverted. 
(c) A typical example of a function $Q$ satisfying the assumptions is

$$
Q(f)=f+f^{1+1 / k}, f \geq 0,
$$

with $0<k<7 / 2$ which leads to a steady state of polytropic form (1.2). More generally, if an isotropic steady state $\left(f_{0}, U_{0}\right)$ is given with $f_{0}$ of the form

$$
f_{0}(x, v)=\phi(E)
$$

with some function $\phi$ then the above assumptions for the Casimir functional hold, if $\phi(E)$ vanishes for $E$ larger than some cut-off energy $E_{0}$, $\phi(E) \leq C\left(E_{0}-E\right)^{k}, E \leq E_{0}$, where $0<k<7 / 2$, and $\phi^{\prime}(E)<0, E<E_{0}$. The existence of a cut-off energy is necessary in order that the steady state has finite mass. The growth condition is essential for the compactness properties of $\mathcal{H}$; cf. the difficulties in the limiting case $k=7 / 2$, and note also that the polytropic ansatz with $k>7 / 2$ leads to steady states with infinite mass. Finally, it is generally believed that steady states are unstable if the monotonicity condition on $\phi$ is violated sufficiently strongly. In this sense one can say that the assumptions on $Q$ are optimal.

(d) The function

$$
Q(f)=\left\{\begin{array}{cl}
f & , 0 \leq f \leq 1, \\
\frac{1}{2}\left(f^{2}+1\right) & , f>1
\end{array}\right.
$$

also satisfies our assumptions and leads to

$$
f_{0}(x, v)=\left\{\begin{array}{cl}
E / E_{0} & , E<E_{0} \\
0 & , E \geq E_{0}
\end{array}\right.
$$

with some $E_{0}<0$. Thus the fact that we do not require $Q \in C^{2}(] 0, \infty[)$ with $Q^{\prime \prime}>0$ allows for examples where $f_{0}$ has jump discontinuities, and these steady states will turn out to be dynamically stable as well.

We collect some estimates for $\rho_{f}$ and $U_{f}$ induced by an element $f \in \mathcal{F}_{M}$. As in the rest of the paper constants denoted by $C$ are positive, may depend on $Q$ and $M$, and their value may change from line to line.

Lemma 1 Let $n:=k+3 / 2$ so that $1+1 / n>6 / 5$. Then for any $f \in \mathcal{F}_{M}$ the following holds: 
(a) $f \in L^{1+1 / k}\left(\mathbb{R}^{6}\right)$ with

$$
\iint f^{1+1 / k} d v d x+\iint f d v d x \leq C
$$

(b) $\rho_{f} \in L^{1+1 / n}\left(\mathbb{R}^{3}\right)$ with

$$
\begin{aligned}
\int \rho_{f}^{1+1 / n} d x & \leq C\left[\iint f^{1+1 / k} d v d x\right]^{k / n}\left[\iint|v|^{2} f d v d x\right]^{(n-k) / n} \\
& \leq C E_{\mathrm{kin}}(f)^{\frac{3}{2 n}}
\end{aligned}
$$

(c) $U_{f} \in L^{6}\left(\mathbb{R}^{3}\right)$ with $\nabla U_{f} \in L^{2}\left(\mathbb{R}^{3}\right)$, the two forms of $E_{\text {pot }}(f)$ stated above are equal, and

$$
\int\left|\nabla U_{f}\right|^{2} d x \leq C\left\|\rho_{f}\right\|_{6 / 5}^{2} \leq C E_{\mathrm{kin}}(f)^{1 / 2}
$$

The assertions in (b) and (c) remain valid in the limiting case $k=7 / 2$ where $n=5$, cf. Remark (a) above.

Proof. Part (a) is obvious from assumption (Q1). Splitting the $v$-integral according to $|v| \leq R$ and $|v|>R$ and optimizing in $R$ yields

$$
\rho_{f}^{1+1 / n} \leq C\left(\int f^{1+1 / k} d v\right)^{k / n}\left(\int|v|^{2} f d v\right)^{(n-k) / n} .
$$

Therefore, the first estimate in (b) follows from Hölder's inequality with indices $n / k$ and $n /(n-k)$, and part (a) implies the second estimate in (b). Since $\rho_{f} \in L^{1} \cap L^{1+1 / n}\left(\mathbb{R}^{3}\right)$ and $1+1 / n>6 / 5$ we find by interpolation,

$$
\int \rho_{f}^{6 / 5} \leq C E_{\mathrm{kin}}(f)^{3 / 10}
$$

in the limiting case this follows directly without interpolation. The estimates for $U_{f}$ follow from the generalized Young's inequality, and the equality of the two representations for $E_{\text {pot }}(f)$ follows by integration by parts after regularizing $\rho_{f}$ if necessary.

As an immediate corollary of the lemma above we note that on $\mathcal{F}_{M}$ the total energy $\mathcal{H}$ is bounded from below in such a way that $E_{\text {kin }}$-and thus certain norms of $f$ and $\rho_{f}$-remain bounded along minimizing sequences: 
Lemma 2 There exists a constant $C>0$ such that

$$
\mathcal{H}(f) \geq E_{\text {kin }}(f)-C E_{\text {kin }}(f)^{1 / 2}, f \in \mathcal{F}_{M},
$$

in particular,

$$
h_{M}:=\inf _{\mathcal{F}_{M}} \mathcal{H}>-\infty,
$$

and $E_{\text {kin }}$ is bounded along minimizing sequences of $\mathcal{H}$ in $\mathcal{F}_{M}$.

The behavior of $\mathcal{H}$ and $\mathcal{C}$ under scaling transformations can be used to show that $h_{M}$ is negative and to relate the $h_{M}$ 's for different values of $M$ :

Lemma 3 (a) Let $M>0$. Then $-\infty<h_{M}<0$.

(b) For all $M, \bar{M}>0$,

$$
h_{\bar{M}}=(\bar{M} / M)^{7 / 3} h_{M}
$$

Proof. Given any function $f$, we define a rescaled function $\bar{f}(x, v)=$ $f(a x, b v)$, where $a, b>0$. Then

$$
\mathcal{C}(\bar{f})=\iint Q(f(a x, b v)) d v d x=(a b)^{-3} \mathcal{C}(f)
$$

i. e. $f \in \mathcal{F}_{M}$ iff $\bar{f} \in \mathcal{F}_{\bar{M}}$ where $\bar{M}:=(a b)^{-3} M$. Next

$$
\begin{aligned}
& E_{\mathrm{kin}}(\bar{f})=\frac{1}{2} \iint|v|^{2} f(a x, b v) d v d x=a^{-3} b^{-5} E_{\mathrm{kin}}(f), \\
& E_{\mathrm{pot}}(\bar{f})=-\frac{1}{2} \iiint \int \frac{f(a x, b v) f(a y, b w)}{|x-y|} d w d v d y d x=a^{-5} b^{-6} E_{\mathrm{pot}}(f) .
\end{aligned}
$$

To prove (a) we fix any $f \in \mathcal{F}_{M}$ and let $a=b^{-1}$ so that $\bar{f} \in \mathcal{F}_{M}$ as well. Then

$$
\mathcal{H}(\bar{f})=b^{-2} E_{\text {kin }}(f)+b^{-1} E_{\text {pot }}(f)<0
$$

for $b>0$ sufficiently large, since $E_{\text {pot }}(f)<0$. To prove (b) choose $a$ and $b$ such that $a^{-3} b^{-5}=a^{-5} b^{-6}$, i. e., $b=a^{-2}$. Then

$$
\mathcal{H}(\bar{f})=a^{7} \mathcal{H}(f),
$$

and since $a=(\bar{M} / M)^{1 / 3}$ and the mapping $\mathcal{F}_{M} \rightarrow \mathcal{F}_{\bar{M}}, f \mapsto \bar{f}$ is one-to-one and onto this proves (b).

One should note that both Lemma 2 and Lemma 3 remain valid in the limiting case $k=7 / 2$. 


\section{Existence of minimizers for $k<7 / 2$}

It is conceivable that along a minimizing sequence the mass could run off to spatial infinity and/or spread uniformly in space. The main problem in proving the existence of a minimizer is to show that this does not happen, which is done in the next lemma. Combined with a local compactness result for the induced fields and a new version of the splitting technique developed in our previous papers this will yield the existence of minimizers.

Lemma 4 Let $\left(f_{i}\right) \subset \mathcal{F}_{M}$ be a minimizing sequence of $\mathcal{H}$. Then there exist a sequence $\left(a_{i}\right) \subset \mathbb{R}^{3}$ and $\epsilon_{0}>0, R_{0}>0$ such that

$$
\int_{a_{i}+B_{R_{0}}} \int Q\left(f_{i}\right) d v d x \geq \epsilon_{0}
$$

for all sufficiently large $i \in \mathbb{N}$. Here we define $B_{R}:=\left\{x \in \mathbb{R}^{3}|| x \mid \leq R\right\}$.

Proof. For $R>1$ define

$$
K_{R}(x):=\left\{\begin{array}{cl}
R & ,|x|<1 / R \\
1 /|x| & , 1 / R \leq|x| \leq R \\
0 & ,|x|>R
\end{array}\right.
$$

and

$$
F_{R}(x):=\frac{1}{|x|} \mathbf{1}_{\{|x|>R\}}(x), G_{R}(x):=\left(\frac{1}{|x|}-R\right) \mathbf{1}_{\{|x|<1 / R\}}(x)
$$

so that we split the kernel

$$
\frac{1}{|x|}=K_{R}(x)+F_{R}(x)+G_{R}(x), x \in \mathbb{R}^{3} .
$$

Here $\mathbf{1}_{A}$ denotes the indicator function of the set $A$. We split

$$
\frac{1}{4 \pi} \int\left|\nabla U_{i}\right|^{2} d x=\iint \frac{\rho_{i}(x) \rho_{i}(y)}{|x-y|} d y d x=I_{1}+I_{2}+I_{3}
$$

according to (3.1), where $\rho_{i}:=\rho_{f_{i}}$. Since $\left(\rho_{i}\right)$ is bounded in $L^{1+1 / n}\left(\mathbb{R}^{3}\right)$ and by (Q1) also in $L^{1}\left(\mathbb{R}^{3}\right)$, we find from Lemma 1 (b), using the boundedness 
of the kinetic energy,

$$
\begin{aligned}
\left|I_{1}\right| & \leq R \iint_{|x-y|<R} \rho_{i}(x) \rho_{i}(y) d x d y \leq R C \sup _{y \in \mathbb{R}^{3}} \int_{y+B_{R}} \rho_{i}(x) d x \\
& \leq R^{(n+4) /(n+1)} C \sup _{y \in \mathbb{R}^{3}}\left[\int_{y+B_{R}} \rho_{i}^{1+1 / n} d x\right]^{n /(n+1)} \\
& \leq R^{(n+4) /(n+1)} C \sup _{y \in \mathbb{R}^{3}}\left[\int_{y+B_{R}} \int f_{i}^{1+1 / k} d v d x\right]^{k /(n+1)},
\end{aligned}
$$

and

$$
\begin{aligned}
& \left|I_{2}\right| \leq \frac{1}{R} \iint \rho_{i}(x) \rho_{i}(y) d x d y \leq C R^{-1} \\
& \left|I_{3}\right| \leq\left\|\rho_{i}\right\|_{1+1 / n}\left\|\rho_{i} * G_{R}\right\|_{n+1} \leq C\left\|G_{R}\right\|_{(n+1) / 2} \leq C R^{-(5-n) /(n+1)}
\end{aligned}
$$

for the last estimate we used Hölder's and Young's inequality. Since $\left(f_{i}\right)$ is a minimizing sequence we have, for any $R>1$,

$$
h_{M} / 2>\mathcal{H}\left(f_{i}\right) \geq-\left|I_{1}\right|-\left|I_{2}\right|-\left|I_{3}\right|,
$$

provided $i$ is sufficiently large. Therefore,

$$
\begin{array}{r}
\liminf _{i \rightarrow \infty}\left[\sup _{y \in \mathbb{R}^{3}} \int_{y+B_{R}} \int f_{i}^{1+1 / k} d v d x\right]^{k /(n+1)}=\liminf _{i \rightarrow \infty}\left|I_{1}\right| R^{-(n+4) /(n+1)} \\
\geq C R^{-(n+4) /(n+1)}\left[-h_{M} / 2-R^{-1}-R^{-(5-n) /(n+1)}\right](3.5)
\end{array}
$$

By Lemma 3 (a) the right hand side of this estimate is positive for $R$ sufficiently large, and the proof is complete.

Lemma 5 Let $\left(\rho_{i}\right) \subset L^{1} \cap L^{1+1 / n}\left(\mathbb{R}^{3}\right)$ be bounded with respect to both norms and $\rho_{0} \in L^{1} \cap L^{1+1 / n}\left(\mathbb{R}^{3}\right)$ with

$$
\rho_{i} \rightarrow \rho_{0} \text { weakly in } L^{1+1 / n}\left(\mathbb{R}^{3}\right) .
$$

Then for any $R>0$,

$$
\nabla U_{\mathbf{1}_{B_{R}} \rho_{i}} \rightarrow \nabla U_{\mathbf{1}_{B_{R}} \rho_{0}} \text { strongly in } L^{2}\left(\mathbb{R}^{3}\right) .
$$


Proof. Take any $R^{\prime}>R$. Since by assumption on $k$ we have $1+1 / n \in$ ]6/5,5/3[, the mapping

$$
L^{1+1 / n}\left(\mathbb{R}^{3}\right) \ni \rho \mapsto \mathbf{1}_{B_{R^{\prime}}} \nabla U_{\rho} \in L^{2}\left(B_{R^{\prime}}\right)
$$

is compact. Thus the asserted strong convergence holds on $B_{R^{\prime}}$. On the other hand,

$$
\int_{|x| \geq R^{\prime}}\left|\nabla U_{\mathbf{1}_{B_{R}} \rho_{i}}\right|^{2} d x \leq \frac{C}{R^{\prime}-R}\left\|\rho_{i}\right\|_{1}^{2} \leq \frac{C}{R^{\prime}-R}, i \in \mathbb{N} \cup\{0\},
$$

which is arbitrarily small for $R^{\prime}$ large.

We are now ready to show the existence of a minimizer of $\mathcal{H}$.

Theorem 1 Let $M>0$. Let $\left(f_{i}\right) \subset \mathcal{F}_{M}$ be a minimizing sequence of $\mathcal{H}$. Then there is a minimizer $f_{0} \in \mathcal{F}_{M}$, a subsequence (still denoted by $\left(f_{i}\right)$ ), and a sequence of translations $T_{i} f_{i}(x, v)=f_{i}\left(x+a_{i}, v\right)$ with $\left(a_{i}\right) \subset \mathbb{R}^{3}$, such that

$$
\mathcal{H}\left(f_{0}\right)=\inf _{\mathcal{F}_{M}} \mathcal{H}=h_{M}
$$

and $T_{i} f_{i} \rightarrow f_{0}$ weakly in $L^{1+1 / k}\left(\mathbb{R}^{6}\right)$. For the induced potentials we have $\nabla U_{T_{i} f_{i}} \rightarrow \nabla U_{0}$ strongly in $L^{2}\left(\mathbb{R}^{3}\right)$.

Remark. Without admitting shifts in $x$-space the assertion of the theorem is wrong: Starting from a given minimizer $f_{0}$ and a sequence of shift vectors $\left(a_{i}\right) \in \mathbb{R}^{3}$ the sequence $\left(T_{i} f_{0}\right)$ is minimizing and in $\mathcal{F}_{M}$, but if $\left|a_{i}\right| \rightarrow \infty$ this minimizing sequence converges weakly to zero, which is not in $\mathcal{F}_{M}$.

Proof of Theorem 1. Let $\left(f_{i}\right)$ be a minimizing sequence and $\left(a_{i}\right) \subset \mathbb{R}^{3}$ such that the assertion of Lemma 1 holds. Since $\mathcal{H}$ is translation invariant $\left(T_{i} f_{i}\right)$ is again a minimizing sequence. By Lemma 1 $(\mathrm{a}),\left(T_{i} f_{i}\right)$ is bounded in $L^{1+1 / k}\left(\mathbb{R}^{6}\right)$. Thus there exists a weakly convergent subsequence, denoted by $\left(T_{i} f_{i}\right)$ again:

$$
T_{i} f_{i} \rightarrow f_{0} \text { weakly in } L^{1+1 / k}\left(\mathbb{R}^{6}\right) .
$$

Clearly, $f_{0} \geq 0$ a. e. By Lemma 2, $\left(E_{\text {kin }}\left(T_{i} f_{i}\right)\right)$ is bounded so by Lemma 1, $\left(\rho_{i}\right)=\left(\rho_{T_{i} f_{i}}\right)$ is bounded in $L^{1+1 / n}\left(\mathbb{R}^{3}\right)$, and by assumption (Q1) this sequence is also bounded in $L^{1}\left(\mathbb{R}^{3}\right)$. After extracting a further subsequence

$$
\rho_{i} \rightarrow \rho_{0}:=\rho_{f_{0}} \text { weakly in } L^{1+1 / n}\left(\mathbb{R}^{3}\right) .
$$


Also by weak convergence

$$
E_{\text {kin }}\left(f_{0}\right) \leq \liminf _{i \rightarrow \infty} E_{\text {kin }}\left(T_{i} f_{i}\right)<\infty .
$$

By (Q2) the functional $\mathcal{C}$ is convex. Thus by Mazur's Lemma and Fatou's Lemma

$$
\mathcal{C}\left(f_{0}\right) \leq \limsup _{i \rightarrow \infty} \mathcal{C}\left(T_{i} f_{i}\right)=M,
$$

in particular, $\rho_{0} \in L^{1}\left(\mathbb{R}^{3}\right)$ by $(\mathrm{Q} 1)$.

The key step is to show that up to a subsequence we have

$$
\left\|\nabla U_{T_{i} f_{i}}-\nabla U_{0}\right\|_{2} \rightarrow 0
$$

For $R_{0}<R$ we denote $B_{R_{0}, R}:=\left\{x \in \mathbb{R}^{3}\left|R_{0} \leq\right| x \mid \leq R\right\}$, and we split $T_{i} f_{i}$ as follows:

$$
\begin{aligned}
T_{i} f_{i} & =T_{i} f_{i} \mathbf{1}_{B_{R_{0}} \times \mathbb{R}^{3}}+T_{i} f_{i} \mathbf{1}_{B_{R_{0}, R} \times \mathbb{R}^{3}}+T_{i} f_{i} \mathbf{1}_{B_{R, \infty} \times \mathbb{R}^{3}} \\
& =: f_{i}^{1}+f_{i}^{2}+f_{i}^{3} .
\end{aligned}
$$

Due to Lemma 5, $\nabla U_{f_{i}^{1}+f_{i}^{2}}$ converges strongly in $L^{2}$ for any fixed $R$. It thus suffices to show that for any $\epsilon>0$,

$$
\liminf _{i \rightarrow \infty} \int\left|\nabla U_{f_{i}^{3}}\right|^{2} d x<\epsilon
$$

for sufficiently large $R$. By Lemma 1 (b) we only need to show that

$$
\liminf _{i \rightarrow \infty} \iint Q\left(f_{i}^{3}\right) d v d x<\epsilon
$$

for sufficiently large $R$. We use the method of splitting to verify (3.9). According to (3.7),

$$
\begin{aligned}
\mathcal{H}\left(T_{i} f_{i}\right)= & \mathcal{H}\left(f_{i}^{1}\right)+\mathcal{H}\left(f_{i}^{2}\right)+\mathcal{H}\left(f_{i}^{3}\right) \\
& -\iint \frac{\rho_{i}^{2}(x)\left(\rho_{i}^{1}+\rho_{i}^{3}\right)(y)}{|x-y|} d x d y-\iint \frac{\rho_{i}^{1}(x) \rho_{i}^{3}(y)}{|x-y|} d x d y \\
=: & \mathcal{H}\left(f_{i}^{1}\right)+\mathcal{H}\left(f_{i}^{2}\right)+\mathcal{H}\left(f_{i}^{3}\right)-I_{1}-I_{2},
\end{aligned}
$$

with obvious definitions for $\rho_{i}^{1}, \rho_{i}^{2}, \rho_{i}^{3}$. The boundedness of $\left\|\nabla U_{\rho_{i}^{1}+\rho_{i}^{3}}\right\|_{2}$ implies that

$$
I_{1} \leq C\left\|\nabla U_{\rho_{i}^{2}}\right\|_{2}
$$


Since $\rho_{i}^{2}$ converges weakly in $L^{1+1 / n}$ to $\rho_{0}^{2}:=\rho_{0} \mathbf{1}_{B_{R_{0}, R}}$,

$$
\left\|\nabla U_{\rho_{i}^{2}}-\nabla U_{\rho_{0}^{2}}\right\|_{2} \rightarrow 0, i \rightarrow \infty
$$

by Lemma 5. For $R>2 R_{0}$ we use Hölder's inequality to estimate $I_{2}$ as follows:

$$
I_{2} \leq 2 \int_{B_{R_{0}}} \rho_{i}(x) d x \int_{B_{R, \infty}}|y|^{-1} \rho_{i}(y) d y \leq C\left\|\rho_{i}\right\|_{6 / 5}^{2}\left(\frac{R_{0}}{R}\right)^{1 / 2} .
$$

It is a simple calculus exercise to show that

$$
\tau^{7 / 3}+(1-\tau)^{7 / 3} \leq 1-\frac{7}{3} \tau(1-\tau), \tau \in[0,1] .
$$

With Lemma 3 and obvious definitions of $M_{i}^{1}, M_{i}^{2}, M_{i}^{3}$ this implies that

$$
\begin{aligned}
\mathcal{H}\left(f_{i}^{1}\right)+\mathcal{H}\left(f_{i}^{2}\right)+\mathcal{H}\left(f_{i}^{3}\right) & \geq h_{M_{i}^{1}}+h_{M_{i}^{2}}+h_{M_{i}^{3}} \\
& =\left[\left(\frac{M_{i}^{1}}{M}\right)^{7 / 3}+\left(\frac{M_{i}^{2}}{M}\right)^{7 / 3}+\left(\frac{M_{i}^{3}}{M}\right)^{7 / 3}\right] h_{M} \\
& \geq\left[\left(\frac{M_{i}^{1}+M_{i}^{2}}{M}\right)^{7 / 3}+\left(\frac{M_{i}^{3}}{M}\right)^{7 / 3}\right] h_{M} \\
& \geq\left[1-\frac{7}{3} \frac{M_{i}^{1}+M_{i}^{2}}{M} \frac{M_{i}^{3}}{M}\right] h_{M}
\end{aligned}
$$

and thus

$$
\begin{aligned}
h_{M}-\mathcal{H}\left(T_{i} f_{i}\right)-C_{1} h_{M} M_{i}^{1} M_{i}^{3} & \leq I_{i}^{1}+I_{i}^{2} \\
& \leq C_{2}\left[\left\|\nabla U_{f_{0}^{2}}\right\|_{2}+\left\|\nabla U_{\rho_{i}^{2}}-\nabla U_{\rho_{0}^{2}}\right\|_{2}+\left(\frac{R_{0}}{R}\right)^{1 / 2}\right] .
\end{aligned}
$$

Here $R>2 R_{0}$ are so far arbitrary, and the constants $C_{1}, C_{2}$ are independent of $R$ and $R_{0}$. Now assume (3.9) were false. Then there exists $\epsilon_{1}>0$ such that for every $R>0$ and $i$ large we have

$$
\iint Q\left(f_{i}^{3}\right) d v d x \geq \epsilon_{1}
$$

Define

$$
\epsilon_{2}:=-C_{1} h_{M} \epsilon_{0} \epsilon_{1}>0
$$


where $\epsilon_{0}$ is as in Lemma 国, and increase $R_{0}$ from that lemma such that $C_{2}\left\|\nabla U_{f_{0}^{2}}\right\|_{2} \leq \epsilon_{2} / 4$. Next choose $R>2 R_{0}$ such that $C_{2}\left(R_{0} / R\right)^{1 / 2} \leq \epsilon_{2} / 4$. Then for $i$ large,

$$
\begin{aligned}
h_{M}-\mathcal{H}\left(T_{i} f_{i}\right)+\epsilon_{2} & \leq h_{M}-\mathcal{H}\left(T_{i} f_{i}\right)-C_{1} h_{M} M_{i}^{1} M_{i}^{3} \\
& \leq \frac{1}{2} \epsilon_{2}+C_{2}\left\|\nabla U_{\rho_{i}^{2}}-\nabla U_{\rho_{0}^{2}}\right\|_{2} .
\end{aligned}
$$

By (3.11) this contradicts the fact that $\left(T_{i} f_{i}\right)$ is minimizing. Thus (3.9) holds, and (3.6) follows.

Clearly we have $\mathcal{H}\left(f_{0}\right) \leq \lim _{i} \mathcal{H}\left(T_{i} f_{i}\right)$, and it remains to show that $\mathcal{C}\left(f_{0}\right)=$ $M$. Assume that $M_{0}:=\mathcal{C}\left(f_{0}\right)<M ; M_{0}>0$ since otherwise $f_{0}=0$ in contradiction to $\mathcal{H}\left(f_{0}\right)<0$. Let

$$
b:=\left(\frac{M_{0}}{M}\right)^{2 / 3}<1, a:=b^{-1 / 2},
$$

so that by (2.5), $\bar{f}_{0} \in \mathcal{F}_{M}$. Then by (2.6),

$$
\mathcal{H}\left(\bar{f}_{0}\right)=a^{7} \mathcal{H}\left(f_{0}\right)=b^{-7 / 2} h_{M}<h_{M},
$$

a contradiction; recall that $b<1$ and $h_{M}<0$.

\section{Properties of minimizers}

The purpose of the present section is to show that the minimizers obtained in the previous one are indeed steady states of the Vlasov-Poisson system.

Theorem 2 Let $f_{0} \in \mathcal{F}_{M}$ be a minimizer of $\mathcal{H}$. Then

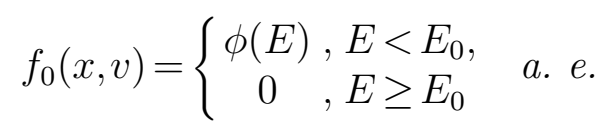

where

$$
\begin{gathered}
E:=\frac{1}{2}|v|^{2}+U_{0}(x), \\
E_{0}:=\lambda_{0} Q^{\prime}(0), \lambda_{0}:=\frac{\iint E f_{0} d v d x}{\iint Q^{\prime}\left(f_{0}\right) f_{0} d v d x}<0,
\end{gathered}
$$

$U_{0}$ is the potential induced by $f_{0}$, and

$$
\phi(E):=\inf \left\{f \geq 0 \mid Q^{\prime}(f)=E / \lambda_{0}\right\}, E \leq E_{0} .
$$

In particular, $f_{0}$ is a steady state of the Vlasov-Poisson system. 


\section{Remark.}

(a) The Euler-Lagrange equation for our constrained minimization problem will give us the relation

$$
\lambda_{0} Q^{\prime}\left(f_{0}\right)=E \text { on } f_{0}^{-1}(] 0, \infty[),
$$

which we want to invert by means of the function $\phi$. Clearly, if $Q^{\prime}$ is strictly increasing then

$$
\phi(E)=\left(Q^{\prime}\right)^{-1}\left(E / \lambda_{0}\right), E \leq E_{0}
$$

(b) Under our general assumption on $Q$ the function $Q^{\prime}:\left[0, \infty\left[\rightarrow\left[Q^{\prime}(0), \infty[\right.\right.\right.$ is continuous, increasing, and onto. This implies that for every $\eta \geq$ $Q^{\prime}(0)$ the set $\left(Q^{\prime}\right)^{-1}(\eta)$ is a closed, bounded interval, and there exists an at most countable set $V_{\text {crit }}$ such that $\left(Q^{\prime}\right)^{-1}(\eta)$ consists of one point for $\eta \notin V_{\text {crit }}$. The function $\phi$ is decreasing with $\left.\left.\phi(]-\infty, E_{0}\right]\right)=[0, \infty[$, and for $f \in\left[0, \infty\left[\right.\right.$ with $\lambda_{0} Q^{\prime}(f) \notin V_{\text {crit }}$ we have $\phi\left(\lambda_{0} Q^{\prime}(f)\right)=f$ as desired.

(c) In the example given by (2.3),

$$
Q^{\prime}(f)=\left\{\begin{array}{l}
1,0 \leq f \leq 1 \\
f, f>1
\end{array}\right.
$$

which is not one-to-one on $[0, \infty[$, but the Euler-Lagrange equation can be inverted to yield (2.4).

Proof of Theorem 2. Let $f_{0}$ and $U_{0}$ be a pointwise defined representative of a minimizer of $\mathcal{H}$ in $\mathcal{F}_{M}$ and its induced potential respectively; to derive the Euler-Lagrange relation we will argue first on $f_{0}^{-1}(] 0, \infty[)$ and then on the complement.

For $\epsilon>0$ small,

$$
K_{\epsilon}:=\left\{(x, v) \in \mathbb{R}^{6} \mid \epsilon \leq f_{0}(x, v) \leq \frac{1}{\epsilon}\right\}
$$

defines a set of positive, finite measure. Let $w \in L^{\infty}\left(\mathbb{R}^{6}\right)$ be compactly supported and non-negative outside $K_{\epsilon}$, and define

$$
G(\sigma, \tau):=\iint Q\left(f_{0}+\sigma \mathbf{1}_{K_{\epsilon}}+\tau w\right) d v d x
$$


for $\tau$ and $\sigma$ close to zero, $\tau \geq 0$, the function $f_{0}+\sigma \mathbf{1}_{K_{\epsilon}}+\tau w$ is bounded on $K_{\epsilon}$, and non-negative otherwise. Therefore, $G$ is continuously differentiable for such $\tau$ and $\sigma$, and $G(0,0)=M$. Since

$$
\partial_{\sigma} G(0,0)=\iint_{K_{\epsilon}} Q^{\prime}\left(f_{0}\right) d v d x \neq 0
$$

there exists by the implicit function theorem a continuously differentiable function $\tau \mapsto \sigma(\tau)$ with $\sigma(0)=0$, defined for $\tau \geq 0$ small, such that $G(\sigma(\tau), \tau)=M$. Hence $f_{0}+\sigma(\tau) \mathbf{1}_{K_{\epsilon}}+\tau w \in \mathcal{F}_{M}$. Furthermore,

$$
\sigma^{\prime}(0)=-\frac{\partial_{\tau} G(0,0)}{\partial_{\sigma} G(0,0)}=-\frac{\iint Q^{\prime}\left(f_{0}\right) w}{\iint_{K_{\epsilon}} Q^{\prime}\left(f_{0}\right)}
$$

Since $G(\sigma(\tau), \tau)$ attains its minimum at $\tau=0$, Taylor expansion implies

$$
0 \leq \mathcal{H}\left(f_{0}+\sigma(\tau) \mathbf{1}_{K_{\epsilon}}+\tau w\right)-\mathcal{H}\left(f_{0}\right)=\tau \iint E\left[\sigma^{\prime}(0) \mathbf{1}_{K_{\epsilon}}+w\right] d v d x+o(\tau)
$$

for $\tau \geq 0$ small. With $(4.1)$ we get

$$
\iint\left[-\lambda_{\epsilon} Q^{\prime}\left(f_{0}\right)+E\right] w d v d x \geq 0
$$

where

$$
\lambda_{\epsilon}:=\frac{\iint_{K_{\epsilon}} E}{\iint_{K_{\epsilon}} Q^{\prime}\left(f_{0}\right)} .
$$

By our choice for $w$ this implies that $E=\lambda_{\epsilon} Q^{\prime}\left(f_{0}\right)$ a. e. on $K_{\epsilon}$ and $E \geq$ $\lambda_{\epsilon} Q^{\prime}\left(f_{0}\right)$ otherwise. This shows that $\lambda_{\epsilon}=\lambda_{0}$ does in fact not depend on $\epsilon$. Letting $\epsilon \rightarrow 0$, we conclude that

$$
\begin{aligned}
& E=\lambda_{0} Q^{\prime}\left(f_{0}\right) \text { a. e. on } f_{0}^{-1}(] 0, \infty[), \\
& E \geq \lambda_{0} Q^{\prime}(0)=E_{0} \text { a. e. on } f_{0}^{-1}(0) .
\end{aligned}
$$

If we multiply (4.3) by $f_{0}$ and integrate we obtain the asserted formula for $\lambda_{0}$, and $\lambda_{0}<0$ as claimed, since

$$
\iint E f_{0} d v d x=E_{\text {kin }}\left(f_{0}\right)-2 E_{\text {pot }}\left(f_{0}\right)<\mathcal{H}\left(f_{0}\right)<0
$$

We need to invert (4.3). Let $V_{\text {crit }}$ be the at most countable set of values where $Q^{\prime}$ is not one-to-one, cf. part (a) of the remark above. Since for any 
constant $\eta \in \mathbb{R}$ the set $E^{-1}(\eta)$ has measure zero-for fixed $x$ this is a sphere in $v$-space - we conclude that

$$
S_{\text {crit }}:=\left\{(x, v) \in \mathbb{R}^{6} \mid E(x, v) / \lambda_{0} \in V_{\text {crit }}\right\}
$$

is a set of measure zero, and on $f_{0}^{-1}(] 0, \infty[) \backslash S_{\text {crit }}$ the Euler-Lagrange equation (4.3) can be inverted to yield

$$
f_{0}(x, v)=\phi(E)
$$

as claimed, cf. part (a) of the remark above. Together with (4.4) this proves that $f_{0}$ is a. e. equal to a function of the particle energy $E$.

Next we study the regularity, symmetry, and uniqueness of minimizers. Let $C_{c}^{m}$ and $C_{b}^{m}$ denote the space of $C^{m}$ functions with compact support and with bounded derivatives up to order $m$, respectively.

Theorem 3 (a) Let $f_{0} \in \mathcal{F}_{M}$ be a minimizer of $\mathcal{H}$. Then $f_{0}$ is spherically symmetric with respect to some point in $x$-space.

(b) If $k \geq 1 / 2$ assume in addition that

$$
\phi(E) \leq C(-E)^{k}, E \rightarrow-\infty,
$$

where $\phi$ is defined by $Q$ as in Theorem Q; this condition is compatible with the general assumptions on $Q$. Then $U_{0} \in C_{b}^{2}\left(\mathbb{R}^{3}\right)$ with $\lim _{|x| \rightarrow \infty} U_{0}(x)=0$ and $\rho_{0} \in C_{c}^{1}\left(\mathbb{R}^{3}\right)$.

(c) If in particular $Q(f)=f+f^{1+1 / k}, f \geq 0$, with $0<k<7 / 2$ then up to a shift in $x$-space there are at most two minimizers of $\mathcal{H}$ in $\mathcal{F}_{M}$.

Proof. To prove the spherical symmetry of $f_{0}$ we denote by $f_{0}^{*}$ the spherically symmetric rearrangement of $f_{0}$ with respect to $x$. The rearrangement does not change the kinetic energy, nor the Casimir functional. By [13, Thm. 3.7] it can only decrease the potential energy, more precisely,

$$
-\iint \frac{f_{0}(x, v) f_{0}(y, w)}{|x-y|} d x d y \geq-\iint \frac{f_{0}^{*}(x, v) f_{0}^{*}(y, w)}{|x-y|} d x d y
$$

for almost all $v, w \in \mathbb{R}^{3}$. But since $f_{0}$ already minimizes $\mathcal{H}, f_{0}^{*}$ minimizes $\mathcal{H}$ as well and the potential energy remains unchanged under the rearrangement 
which implies that in the above, equality holds for almost all $v, w \in \mathbb{R}^{3}$. By [13, Thm. 3.9] this can happen only if $f_{0}(x, v)=f_{0}^{*}\left(x+T_{v}, v\right)$ for some possibly $v$-dependent shift vector $T_{v}$. Since both $f_{0}$ and $f_{0}^{*}$ are minimizers they are both of the form stated in Theorem 2, so $f_{0}(x, v)=\phi\left(E_{0}-\frac{1}{2}|v|^{2}-U_{f_{0}}(x)\right)$ and $f_{0}^{*}(x, v)=\phi\left(E_{0}^{*}-\frac{1}{2}|v|^{2}-U_{f_{0}^{*}}(x)\right)$. The explicit form of $E_{0}$ now implies that $E_{0}=E_{0}^{*}$, hence $U_{f_{0}}(x)=U_{f_{0}^{*}}\left(x+T_{v}\right)$ and the translation $T_{v}$ is independent of $v$. Hence the minimizer $f_{0}$ is a spatial translation of $f_{0}^{*}$ which proves the symmetry assertion. In passing we note that the symmetry can also be obtained without the rearrangement concept, cf. 18.

To prove part (b), consider first the case where $k<1 / 2$ i. e., $n<2$. Then $\rho_{0} \in L^{p} \cap L^{1}$ with $p=1+1 / n>3 / 2$. The usual $L^{p}$-regularity theory and Sobolev's embedding theorem implies that

$$
U_{0} \in W_{\text {loc }}^{2, p}\left(\mathbb{R}^{3}\right) \subset C\left(\mathbb{R}^{3}\right) .
$$

Moreover, for any $R>0$ and $x \in \mathbb{R}^{3}$,

$$
\begin{aligned}
-U_{0}(x) & =\int_{|x-y|<1 / R} \frac{\rho_{0}(y)}{|x-y|} d y+\int_{1 / R \leq|x-y|<R} \cdots+\int_{|x-y| \geq R} \cdots \\
& \leq C\left\|\rho_{0}\right\|_{p}\left(\int_{0}^{1 / R} r^{2-q} d r\right)^{1 / q}+R \int_{|y| \geq|x|-R} \rho_{0}(y) d y+\frac{M}{R},
\end{aligned}
$$

where $q$ is the conjugate exponent to $p$, so $q<3$. This implies that

$$
U_{0} \in C_{b}\left(\mathbb{R}^{3}\right), \lim _{|x| \rightarrow \infty} U_{0}(x)=0
$$

This in turn implies that for $|x|$ sufficiently large, $E>E_{0}$; note that the latter quantity is negative by Theorem 2. By the same theorem, $f_{0}$ and $\rho_{0}$ have compact support.

To continue, we note that since $f_{0}$ depends only on the particle energy $E$ via the function $\phi$,

$$
\rho_{0}(x)=h_{\phi}\left(U_{0}(x)\right), x \in \mathbb{R}^{3}
$$

where

$$
h_{\phi}(u):=4 \pi \sqrt{2} \int_{u}^{\infty} \phi(E) \sqrt{E-u} d E, u \in \mathbb{R}
$$

note that $h_{\phi}(u)=0$ for $u \geq E_{0}$. By the general assumptions on $Q$ the function $h_{\phi}$ is continuously differentiable. Thus the regularity of $U_{0}$ implies that $\rho_{0} \in$ 
$C_{c}\left(\mathbb{R}^{3}\right)$, this in turn implies that $U_{0} \in C_{b}^{1}\left(\mathbb{R}^{3}\right)$, thus $\rho_{0} \in C_{c}^{1}\left(\mathbb{R}^{3}\right)$, and finally $U_{0} \in C_{b}^{2}\left(\mathbb{R}^{3}\right)$.

Consider now the case that $k \geq 1 / 2$. Clearly we are done if we can but prove that $\rho_{0}$ is not only in $L^{p}$ with $p=1+1 / n$, which is now to small for the argument above, but in some $L^{p}$ with $p>3 / 2$. To show this, we use a bootstrap argument, based on (4.5). For this to work, we need some control on the growth of the function $h_{\phi}$ which is the reason for the extra assumption on $\phi$. Indeed, under that assumption the following estimate holds:

$$
h_{\phi}(u) \leq C\left(1+\left(E_{0}-u\right)^{n}\right), u \leq E_{0} .
$$

If we use this estimate on the set where $\rho_{0}$ is large - this set has finite measure - and the integrability of $\rho_{0}$ on the complement we find that

$$
\int \rho_{0}(x)^{p} d x \leq C+\int\left(-U_{0}(x)\right)^{n p} d x .
$$

If we would pick the limiting case $n=5$, i. e., $\rho_{0} \in L^{6 / 5}$, we would find by Young's inequality that $U_{0} \in L^{6}$, and bootstrapping this via (4.7) gives us $\rho_{0} \in$ $L^{6 / 5}$ back. However, for $n<5$ this works better: Starting with $p_{0}=1+1 / n$ we apply Young's inequality to find that $U_{0}$ lies in $L^{q}$ with $q=\left(1 / p_{0}-2 / 3\right)^{-1}>1$, and substituting this into (4.7) we conclude that $\rho_{0} \in L^{p_{1}}$ with $p_{1}=q / n$; note that by assumption $p_{0}<3 / 2$. If $p_{1}>3 / 2$ we are done. If $p_{1}=3 / 2$ we decrease $p_{1}$ slightly - note that $\rho_{0} \in L^{1}$ - so that in the next bootstrap step we find $p_{2}$ as large as we wish. If $p_{1}<3 / 2$ we repeat the process. By induction one sees that

$$
p_{k}=\frac{3(1+1 / n)(n-1)}{n^{k}(n-5)+2 n+2}>1
$$

as long as $p_{k-1}<3 / 2$. But since $2 \leq n<5$ the denominator would eventually become negative so that the process must stop after finitely many steps.

As to part (c) we first observe that up to some shift $U_{0}$ as a function of the radial variable $r:=|x|$ solves the equation

$$
\frac{1}{r^{2}}\left(r^{2} U_{0}^{\prime}\right)^{\prime}=c_{k}\left(E_{0}-U_{0}\right)_{+}^{k+3 / 2}, r>0,
$$

with some appropriately defined constant $c_{k}$. Here' denotes the derivative with respect to $r$. The assertion now follows from the scaling properties of (4.8), and we refer to [8, Thm. 3] for the details. 


\section{Dynamical stability}

Let $f_{0} \in \mathcal{F}_{M}$ be a minimizer as obtained in Theorem 11. To investigate its dynamical stability we note first that

$$
\begin{aligned}
\mathcal{H}(f)-\mathcal{H}\left(f_{0}\right) & =\iint\left(\frac{1}{2}|v|^{2}+U_{0}\right)\left(f-f_{0}\right) d v d x-\frac{1}{8 \pi}\left\|\nabla U_{f}-\nabla U_{0}\right\|_{2}^{2} \\
& =: d\left(f, f_{0}\right)-\frac{1}{8 \pi}\left\|\nabla U_{f}-\nabla U_{0}\right\|_{2}^{2}, f \in \mathcal{F}_{M} .
\end{aligned}
$$

Since $\mathcal{C}(f)=\mathcal{C}\left(f_{0}\right)$,

$$
d\left(f, f_{0}\right)=\iint\left[E\left(f-f_{0}\right)-\lambda_{0}\left(Q(f)-Q\left(f_{0}\right)\right)\right] d v d x
$$

Since $Q$ is convex and the Lagrange multiplier $\lambda_{0}$ from Theorem 2 is negative, the integrand can be estimated from below by

$$
\left(E-\lambda_{0} Q^{\prime}\left(f_{0}\right)\right)\left(f-f_{0}\right) .
$$

According to Theorem 2, this quantity is zero on $\operatorname{supp} f_{0}$, while on $\mathbb{R}^{6} \backslash$ $\operatorname{supp} f_{0}$ it equals

$$
\left(E-\lambda_{0} Q^{\prime}(0)\right) f=\left(E-E_{0}\right) f \geq 0 .
$$

Thus we see that

$$
d\left(f, f_{0}\right) \geq 0, f \in \mathcal{F}_{M} .
$$

We are now ready to state our stability result. Note that if we shift a minimizer in space we obtain another minimizer. Moreover, we do in general not know whether the minimizers are unique up to spatial shifts. This fact is reflected in two versions of our stability result.

Theorem 4 Let $\mathcal{M}_{M} \subset \mathcal{F}_{M}$ denote the set of all minimizers of $\mathcal{H}$ in $\mathcal{F}_{M}$.

(a) For every $\epsilon>0$ there is a $\delta>0$ such that for any solution $t \mapsto f(t)$ of the Vlasov-Poisson system with $f(0) \in C_{c}^{1}\left(\mathbb{R}^{6}\right) \cap \mathcal{F}_{M}$,

$$
\inf _{f_{0} \in \mathcal{M}_{M}}\left[d\left(f(0), f_{0}\right)+\frac{1}{8 \pi}\left\|\nabla U_{f(0)}-\nabla U_{0}\right\|_{2}^{2}\right]<\delta
$$

implies that

$$
\inf _{f_{0} \in \mathcal{M}_{M}}\left[d\left(f(t), f_{0}\right)+\frac{1}{8 \pi}\left\|\nabla U_{f(t)}-\nabla U_{f_{0}}\right\|_{2}^{2}\right]<\epsilon, t \geq 0 .
$$


(b) Suppose that $f_{0} \in \mathcal{M}_{M}$ is isolated, i. e.,

$$
\inf \left\{\left\|\nabla U_{f_{0}}-\nabla U_{\tilde{f}_{0}}\right\|_{2} \mid \tilde{f}_{0} \in \mathcal{M}_{M} \backslash\left\{T^{a} f_{0} \mid a \in \mathbb{R}^{3}\right\}\right\}>0 .
$$

Then for every $\epsilon>0$ there is a $\delta>0$ such that for any solution $t \mapsto f(t)$ of the Vlasov-Poisson system with $f(0) \in C_{c}^{1}\left(\mathbb{R}^{6}\right) \cap \mathcal{F}_{M}$,

$$
d\left(f(0), f_{0}\right)+\frac{1}{8 \pi}\left\|\nabla U_{f(0)}-\nabla U_{0}\right\|_{2}^{2}<\delta
$$

implies that for every $t \geq 0$ there exists a shift vector $a \in \mathbb{R}^{3}$ such that

$$
d\left(f(t), T^{a} f_{0}\right)+\frac{1}{8 \pi}\left\|\nabla U_{f(t)}-\nabla U_{T^{a} f_{0}}\right\|_{2}^{2}<\epsilon .
$$

Here $T^{a} f(x, v):=f(x+a, v)$ for $a \in \mathbb{R}^{3}$.

\section{Remark.}

(a) By Theorem 3 (c) the assumption of part (b) holds for the polytropes.

(b) We only showed that $d\left(f, f_{0}\right) \geq 0$ for $f \in \mathcal{F}_{M}$, but one may think of this term as a weighted $L^{2}$-difference of $f$ and $f_{0}$. For example, if $Q \in C^{2}(] 0, \infty[)$ with

$$
c_{Q}:=\inf _{0<f \leq f_{\max }} Q^{\prime \prime}(f)>0
$$

where $f_{\max } \geq\left\|f_{0}\right\|_{\infty}$ as would be the case for the polytropes $Q(f)=$ $f+f^{1+1 / k}$ with $1 \leq k<7 / 2$, then by Taylor-expanding $Q$ we find

$$
d\left(f, f_{0}\right) \geq \frac{1}{2} c_{Q}\left\|f-f_{0}\right\|_{2}^{2}, f \in \mathcal{F}_{M} \text { with } f \leq f_{\text {max }}
$$

observe that the size restriction on $f$ propagates along solutions of the Vlasov-Poisson system.

(c) The restriction $f(0) \in \mathcal{F}_{M}$ for the perturbed initial data is acceptable from a physics point of view: A physical perturbation of a given galaxy, say by the gravitational pull of some outside object, would result in a perturbed state which is an equimeasurable rearrangement of the original state, in particular, the value of $\mathcal{C}(f)$ remains unchanged. 
Proof of Theorem 1. Assume the assertion of part (a) were false. Then there exist $\epsilon>0, t_{n}>0$, and $f_{n}(0) \in C_{c}^{1}\left(\mathbb{R}^{6}\right) \cap \mathcal{F}_{M}$ such that for all $n \in \mathbb{N}$,

$$
\inf _{f_{0} \in \mathcal{M}_{M}}\left[d\left(f_{n}(0), f_{0}\right)+\frac{1}{8 \pi}\left\|\nabla U_{f_{n}(0)}-\nabla U_{0}\right\|_{2}^{2}\right]<\frac{1}{n}
$$

but

$$
\inf _{f_{0} \in \mathcal{M}_{M}}\left[d\left(f_{n}\left(t_{n}\right), f_{0}\right)+\frac{1}{8 \pi}\left\|\nabla U_{f_{n}\left(t_{n}\right)}-\nabla U_{0}\right\|_{2}^{2}\right] \geq \epsilon .
$$

By (5.2) and (5.1),

$$
\lim _{n \rightarrow \infty} \mathcal{H}\left(f_{n}(0)\right)=h_{M} .
$$

Since both $\mathcal{H}$ and $\mathcal{C}$ are conserved along classical solutions as launched by $f_{n}(0)$,

$$
\lim _{n \rightarrow \infty} \mathcal{H}\left(f_{n}\left(t_{n}\right)\right)=h_{M} \text { and } f_{n}\left(t_{n}\right) \in \mathcal{F}_{M}, n \in \mathbb{N},
$$

i. e., $\left(f_{n}\left(t_{n}\right)\right)$ is a minimizing sequence for $\mathcal{H}$ in $\mathcal{F}_{M}$. Up to a subsequence we may therefore assume by Theorem 10 that there exists a minimizer $f_{0} \in \mathcal{F}_{M}$ and a sequence $\left(a_{n}\right) \subset \mathbb{R}^{3}$ such that

$$
\left\|\nabla U_{f_{n}\left(t_{n}\right)}-\nabla U_{T^{a_{n} f_{0}}}\right\|_{2}^{2} \rightarrow 0 ;
$$

note that for any $f \in \mathcal{F}_{M}$ and $a \in \mathbb{R}^{3}$,

$$
\left\|\nabla U_{T^{a} f}-\nabla U_{f_{0}}\right\|_{2}=\left\|\nabla U_{f}-\nabla U_{T^{-a} f_{0}}\right\|_{2},
$$

also $d\left(T^{a} f, f_{0}\right)=d\left(f, T^{-a} f_{0}\right)$. Since $\lim _{n \rightarrow \infty} \mathcal{H}\left(f_{n}\left(t_{n}\right)\right)=h_{M}=\mathcal{H}\left(T^{a_{n}} f_{0}\right)$ we conclude by (5.4) and (5.1) that

$$
d\left(f_{n}\left(t_{n}\right), T^{a_{n}} f_{0}\right) \rightarrow 0, n \rightarrow \infty,
$$

and since $T^{a_{n}} f_{0} \in \mathcal{M}_{M}$ we arrive at a contradiction to (5.3). Thus part (a) is established.

Now assume that $f_{0}$ is an isolated minimizer in $\mathcal{F}_{M}$, and define

$$
\delta_{0}:=\frac{1}{8 \pi} \inf \left\{\left\|\nabla U_{f_{0}}-\nabla U_{\tilde{f}_{0}}\right\|_{2} \mid \tilde{f}_{0} \in \mathcal{M}_{M} \backslash\left\{T^{a} f_{0} \mid a \in \mathbb{R}^{3}\right\}\right\}>0 .
$$

Let $\epsilon>0$ arbitrary. In order to find the corresponding $\delta$ we can without loss of generality assume that $\epsilon<\delta_{0} / 4$. Now choose $\delta>0$ according to part (a), without loss of generality $\delta<\epsilon$, and let $f(0) \in C_{c}^{1}\left(\mathbb{R}^{6}\right) \cap \mathcal{F}_{M}$ be such that

$$
d\left(f(0), f_{0}\right)+\frac{1}{8 \pi}\left\|\nabla U_{f(0)}-\nabla U_{0}\right\|_{2}^{2}<\delta .
$$


The function

$$
\begin{aligned}
h(t, a):= & d\left(f(t), T^{a} f_{0}\right)+\frac{1}{8 \pi}\left\|\nabla U_{f(t)}-\nabla U_{T^{a} f_{0}}\right\|_{2}^{2} \\
= & \iint \frac{1}{2}|v|^{2}\left(f(t)-f_{0}\right) d v d x+\frac{1}{8 \pi}\left\|\nabla U_{f(t)}\right\|_{2}^{2}+\frac{3}{8 \pi}\left\|\nabla U_{f_{0}}\right\|_{2}^{2} \\
& +\int U_{T^{a} f_{0}} \rho_{f(t)} d x
\end{aligned}
$$

is continuous, and since the interaction term goes to zero as $|a| \rightarrow \infty$, uniformly on compact time intervals, $\inf _{a \in \mathbb{R}^{3}} h(t, a)$ is also continuous. Now assume that there exists $t>0$ such that

$$
\inf _{a \in \mathbb{R}^{3}} h(t, a) \geq \epsilon
$$

Since at time zero the left hand side is less then $\epsilon$ there exists some $t^{*}>0$ where

$$
\inf _{a \in \mathbb{R}^{3}} h\left(t^{*}, a\right)=\epsilon .
$$

On the other hand, part (a) provides some $f_{0}^{*} \in \mathcal{M}_{M}$ such that

$$
d\left(f\left(t^{*}\right), f_{0}^{*}\right)+\frac{1}{8 \pi}\left\|\nabla U_{f\left(t^{*}\right)}-\nabla U_{f_{0}^{*}}\right\|_{2}^{2}<\epsilon \leq \frac{\delta_{0}}{4} .
$$

By (5.5) and (5.6) together with the non-negativity of $d$,

$$
\frac{1}{8 \pi}\left\|\nabla U_{f_{0}}-\nabla U_{f_{0}^{*}}\right\|_{2}^{2} \leq \frac{\delta_{0}}{2}
$$

and by the definition of $\delta_{0}$ there must exist some $a^{*} \in \mathbb{R}^{3}$ such that $f_{0}^{*}=T^{a^{*}} f_{0}$. But this means that (5.5) contradicts (5.6), and the proof of part (b) is complete.

\section{The case $k=7 / 2$; the Plummer sphere}

In this section we study the so-called Plummer sphere which corresponds to the minimization of $\mathcal{H}$ on the constraint set

$$
\mathcal{F}_{M}:=\left\{f \in L^{9 / 7}\left(\mathbb{R}^{6}\right) \mid f \geq 0, \iint f^{9 / 7} d v d x=M, E_{\mathrm{kin}}(f)<\infty\right\}
$$


i. e., we take $Q(f)=f^{9 / 7}$ which means $k=7 / 2$ and $n=5$. Due to the fact that the scaling transformation

$$
\left(S_{\lambda} f\right)(x, v)=\lambda^{-7} f\left(\lambda^{-4} x, \lambda v\right)
$$

leaves each term in both $\mathcal{H}$ and $\mathcal{C}$ invariant this case poses additional difficulties.

As we noted at the end of Section 2 the assertion of Lemma 2 remains valid so that there exists a minimizing sequence $\left(f_{i}\right)$ of $\mathcal{H}$ in $\mathcal{F}_{M}$. We shall follow the steps in Section 3 to conclude the existence of a minimizer. The key step is to find the analogue of Lemma 4 in the presence of the scaling (6.1) and to deal with compactness issues in the limiting case $\left(\rho_{i}\right) \subset L^{6 / 5}\left(\mathbb{R}^{3}\right)$, cf. Lemma 5. For a function $h=h(x, v) \geq 0$ and a cut-off parameter $N>0$ we define

$$
h_{\mid N}(x, v):=\left\{\begin{array}{cl}
h(x, v) & \text { if } 1 / N \leq h(x, v) \leq N \\
0 & \text { otherwise }
\end{array}\right.
$$

Lemma 6 Let $\left(f_{i}\right) \subset \mathcal{F}_{M}$ be minimizing. Then there exists a sequence $\left(\lambda_{i}\right) \subset$ ] $0, \infty\left[\right.$ such that up to a subsequence the following holds for $S_{i} f_{i}:=S_{\lambda_{i}} f_{i}$ :

(a) For any $\epsilon>0$ there exists some $N>0$ such that for all sufficiently large $i \in \mathbb{N}$,

$$
\left\|S_{i} f_{i}-\left(S_{i} f_{i}\right)_{\mid N}\right\|_{9 / 7}<\epsilon .
$$

(b) There exist a sequence $\left(a_{i}\right) \subset \mathbb{R}^{3}$ and $\epsilon_{0}>0, R_{0}>0$ such that for all sufficiently large $i \in \mathbb{N}$,

$$
\int_{a_{i}+B_{R_{0}}}\left(S_{i} f_{i}\right)^{9 / 7} d v d x \geq \epsilon_{0}
$$

(c) Let $g_{i}(x, v):=T_{i} S_{i} f_{i}(x, v):=\lambda_{i}^{-7} f_{i}\left(\lambda_{i}^{-4} x+a_{i}, \lambda_{i} v\right), \quad g_{i} \rightarrow g_{0}$ weakly in $L^{9 / 7}\left(\mathbb{R}^{6}\right)$ and $\rho_{i}:=\rho_{g_{i}} \rightarrow \rho_{0}$ weakly in $L^{6 / 5}\left(\mathbb{R}^{3}\right)$. Then for any $R>0$ and up to a subsequence, $\nabla U_{\mathbf{1}_{B_{R}} \rho_{i}} \rightarrow \nabla U_{\mathbf{1}_{B_{R}} \rho_{0}}$ strongly in $L^{2}\left(\mathbb{R}^{3}\right)$.

Proof. To prove part (a) we wish to employ the results in [8], so we consider $\left(f_{i}^{*}\right)$, the sequence of spherically symmetric rearrangements with respect to $x$, which is again minimizing and in $\mathcal{F}_{M}$. By [8, Thm. 2] there exists a symmetric minimizer $g$ such that $S_{i} f_{i}^{*} \rightarrow g$ weakly in $L^{9 / 7}\left(\mathbb{R}^{6}\right)$. 
Since $\left\|S_{i} f_{i}^{*}\right\|_{9 / 7}=\left\|f_{i}^{*}\right\|_{9 / 7}=M=\|g\|_{9 / 7}$ it follows that $S_{i} f_{i}^{*} \rightarrow g$ strongly in $L^{9 / 7}\left(\mathbb{R}^{6}\right)$. Note that since $S_{i} f_{i}^{*}$ is spherically symmetric and decreasing in $x$ and equi-measurable with $S_{i} f_{i}$ we have $S_{i} f_{i}^{*}=\left(S_{i} f_{i}\right)^{*}$. Abusing the notation we abbreviate $g_{i}:=S_{i} f_{i}$ in the proofs of parts (a) and (b).

For $\epsilon>0$ we choose $N>0$ such that $\|g\|_{L^{9 / 7}\left(A_{N}\right)}<\epsilon / 2$ where $A_{N}=\{g \leq$ $1 / N \vee g \geq N\}$. Let $A_{i, N}=\left\{g_{i}^{*}<1 / N \vee g_{i}^{*}>N\right\}$. Then for $i$ sufficiently large,

$$
\begin{aligned}
\left\|g_{i}^{*}-g_{i \mid N}^{*}\right\|_{9 / 7} & =\left\|g_{i}^{*}\right\|_{L^{9 / 7}\left(A_{i, N}\right)} \leq\left\|g_{i}^{*}-g\right\|_{L^{9 / 7}\left(A_{i, N}\right)}+\|g\|_{L^{9 / 7}\left(A_{i, N}\right)} \\
& \leq \epsilon / 2+\|g\|_{L^{9 / 7}\left(A_{i, N}\right)} .
\end{aligned}
$$

Up to a subsequence, $g_{i}^{*} \rightarrow g$ pointwise a. e., and thus $\limsup _{i \rightarrow \infty} \mathbf{1}_{A_{i, N}} \leq \mathbf{1}_{A_{N}}$ a. e.. Therefore, by Fatou's lemma we get

$$
\limsup _{i \rightarrow \infty}\|g\|_{L^{9 / 7}\left(A_{i, N}\right)} \leq\|g\|_{L^{9 / 7}\left(A_{N}\right)}<\epsilon / 2 .
$$

Hence up to a subsequence and for $i$ sufficiently large,

$$
\left\|g_{i}^{*}-g_{i \mid N}^{*}\right\|_{9 / 7}<\epsilon \text {. }
$$

Now by the equi-measurability of the rearrangements, (6.2) implies part (a) of the lemma. In fact, for any function $h \geq 0$ and $p \geq 1$,

$$
\begin{aligned}
\int\left(h-h_{\mid N}\right)^{p} & =p \int_{0}^{\infty} s^{p-1} \mu\left\{h \mathbf{1}_{\{h<1 / N \vee h>N\}}>s\right\} d s \\
& =p \int_{0}^{\infty} s^{p-1}[\mu\{s<h<1 / N\}+\mu\{h>\max [N, s]\}] d s \\
& =p \int_{0}^{\infty} s^{p-1}\left[\mu\left\{s<h^{*}<1 / N\right\}+\mu\left\{h^{*}>\max [N, s]\right\}\right] d s \\
& =\int\left(h^{*}-h^{*}{ }_{\mid N}\right)^{p} .
\end{aligned}
$$

Taking $h=g_{i}=S_{i} f_{i}$ and recalling that $S_{i} f_{i}^{*}=\left(S_{i} f_{i}\right)^{*}$ proves part (a) of the lemma.

To prove part (b) we split for $N>0, g_{i}=g_{i \mid N}+\left(g_{i}-g_{i \mid N}\right)$. With the bounded sequence $\left(g_{i \mid N}\right)$ instead of $\left(f_{i}\right)$ we split as in (3.2) of Lemma 1 . Since $\left(g_{i \mid N}\right)$ is bounded in $L^{1} \cap L^{\infty}$ the induced spatial densities are bounded in, say, $L^{5 / 4}$ which we use to bound $I_{3} ; I_{1}$ and $I_{2}$ are treated as before. Thus instead of (3.5) we obtain

$$
\begin{aligned}
{\left[\sup _{y \in \mathbb{R}^{3}} \int_{y+B_{R}} \int\left(g_{i}\right)^{9 / 7}\right.} & d v d x]^{7 / 12} \geq\left[\sup _{y \in \mathbb{R}^{3}} \int_{y+B_{R}} \int\left(g_{i \mid N}\right)^{9 / 7} d v d x\right]^{7 / 12} \\
\geq C R^{-3 / 2} & {\left[-h_{M} / 2-C R^{-1}-C R^{-1 / 5}-\left\|\nabla U_{g_{i}-g_{i \mid N}}\right\|_{2}^{2}\right] . }
\end{aligned}
$$


Because of part (a) and Lemma 1 we can choose $N>0$ such that $\left\|\nabla U_{g_{i}-g_{i \mid N}}\right\|_{2}<-h_{M} / 4$. Then we choose $R>0$ such that the bracket is positive, and the proof of part (b) is complete.

As to part (c) we first show that for any $R>0$ the set $\left\{\nabla U_{\mathbf{1}_{B_{R}} \rho_{i}} \mid i \in \mathbb{N}\right\}$ is relatively compact in $L^{2}\left(\mathbb{R}^{3}\right)$. To see this note that we can for any $\epsilon>0$ choose $N>0$ and $R^{\prime}>R$ such that for $i$ sufficiently large,

$$
\left\|\mathbf{1}_{B_{R^{\prime}}} \nabla U_{\mathbf{1}_{B_{R}} g_{i \mid N}}-\nabla U_{\mathbf{1}_{B_{R}} g_{i}}\right\|_{2}<\epsilon
$$

cf. part (a) together with Lemma 1 and the splitting used in the proof of Lemma 5. For $R^{\prime}$ and $N$ fixed the set $\left\{\mathbf{1}_{B_{R^{\prime}}} \nabla U_{\mathbf{1}_{B_{R}} g_{i \mid N}} \mid i \in \mathbb{N}\right\}$ is relatively compact in $L^{2}\left(\mathbb{R}^{3}\right)$ since $\left\{g_{i \mid N}\right\}$ is bounded in $L^{1} \cap L^{\infty}\left(\mathbb{R}^{6}\right)$ and thus the set of induced densities is bounded, say, in $L^{5 / 4}\left(\mathbb{R}^{3}\right)$. This implies the relative compactness stated above. Next we observe that $\mathbf{1}_{B_{R}} \rho_{i} \rightarrow \mathbf{1}_{B_{R}} \rho_{0}$ weakly in $L^{6 / 5}\left(\mathbb{R}^{3}\right)$ which implies that that $\nabla U_{\mathbf{1}_{B_{R}} \rho_{i}} \rightarrow \nabla U_{\mathbf{1}_{B_{R}} \rho_{0}}$ weakly in $L^{2}\left(\mathbb{R}^{3}\right)$. Together, this yields the assertion of part (c).

We are now ready to prove the analogue of Theorem 1 for the limiting case $k=7 / 2$ :

Theorem 5 Let $M>0$. Let $\left(f_{i}\right) \subset \mathcal{F}_{M}$ be a minimizing sequence of $\mathcal{H}$. Then there is a minimizer $f_{0} \in \mathcal{F}_{M}$, a subsequence (still denoted by $\left(f_{i}\right)$ ), and a sequence of translations and scalings:

$$
T_{i} S_{i} f_{i}(x, v):=\lambda_{i}^{-7} f_{i}\left(\lambda_{i}^{-4} x+a_{i}, \lambda_{i} v\right)
$$

with $\left(a_{i}\right) \subset \mathbb{R}^{3}$ and $\lambda_{i}>0$ such that

$$
\mathcal{H}\left(f_{0}\right)=\inf _{\mathcal{F}_{M}} \mathcal{H}=h_{M}
$$

and $T_{i} S_{i} f_{i} \rightarrow f_{0}$ weakly in $L^{9 / 7}\left(\mathbb{R}^{6}\right)$. For the induced potentials we have $\nabla U_{T_{i} S_{i} f_{i}} \rightarrow \nabla U_{0}$ strongly in $L^{2}\left(\mathbb{R}^{3}\right)$.

Proof. We choose $\left(\lambda_{i}\right),\left(a_{i}\right), \epsilon_{0}>0$, and $R_{0}>0$ according to Lemma 6 and define $g_{i}:=T_{i} S_{i} f_{i}$, which is again a minimizing sequence in $\mathcal{F}_{M}$. This sequence is bounded in $L^{9 / 7}\left(\mathbb{R}^{6}\right)$, and by Lemma 1 (b) the induced spatial densities $\rho_{i}$ are bounded in $L^{6 / 5}\left(\mathbb{R}^{3}\right)$ so that up to a subsequence, $g_{i} \rightarrow f_{0}$ weakly in $L^{9 / 7}\left(\mathbb{R}^{6}\right)$, and $\rho_{i} \rightarrow \rho_{0}$ weakly in $L^{6 / 5}\left(\mathbb{R}^{3}\right)$. We now proceed as in 
the proof of Theorem 1 with $\left(g_{i}\right)$ instead of $\left(f_{i}\right)$, the only difference being that instead of Lemma 4 and 5 we use the corresponding parts of Lemma 6 in order to prove the strong converge of the fields (3.6).

Next we show the analogues of the assertions of Theorems 2 and 3 :

Theorem 6 Let $f_{0} \in \mathcal{F}_{M}$ be a minimizer of $\mathcal{H}$. Then

$$
f_{0}(x, v)=\left\{\begin{array}{cl}
\left(E / \lambda_{0}\right)^{7 / 2} & , E<0 \\
0 & , E \geq 0
\end{array}\right.
$$

with some constant $\lambda_{0}<0$, in particular, $f_{0}$ is a steady state of the VlasovPoisson system. Moreover, $f_{0}$ is spherically symmetric with respect to some point in $x$-space, and up to scalings and translations in $x$,

$$
U_{0}(r)=-c_{0}\left(1+r^{2}\right)^{-1 / 2}, \rho_{0}(r)=\frac{3 c_{0}}{4 \pi}\left(1+r^{2}\right)^{-5 / 2}, r \geq 0,
$$

where the positive constant $c_{0}$ depends on $\lambda_{0}$.

Proof. The identity for $f_{0}$ follows exactly as in the proof of Theorem 2. The spherical symmetry follows as in the proof of Theorem 3. By monotonicity $\left.\left.\lim _{r \rightarrow \infty} U_{0}(r) \in\right]-\infty, 0\right]$ exists, and since $U_{0} \in L^{6}\left(\mathbb{R}^{3}\right)$ this limit must be zero so that $U_{0}$ is a solution of the corresponding Emden-Fowler equation (4.8) with $k=7 / 2$ and $E_{0}=0$. The uniqueness up to scalings follows as in 8 , Thm. 3], and the explicit formulas can be checked by direct computation.

Finally, we state the stability theorem for the limiting case $k=7 / 2$ :

Theorem 7 Let $f_{0} \in \mathcal{F}_{M}$ be a minimizer of $\mathcal{H}$. Then for every $\epsilon>0$ there is a $\delta>0$ such that for any solution $t \mapsto f(t)$ of the Vlasov-Poisson system with $f(0) \in C_{c}^{1} \cap \mathcal{F}_{M}$,

$$
d\left(f(0), f_{0}\right)+\frac{1}{8 \pi}\left\|\nabla U_{f(0)}-\nabla U_{f_{0}}\right\|_{L^{2}}^{2}<\delta
$$

implies that for every $t \geq 0$ there exits a shift vector $a \in \mathbb{R}^{3}$ and a scaling parameter $\lambda>0$ such that

$$
d\left(f(t), T^{a} S_{\lambda} f_{0}\right)+\frac{1}{8 \pi}\left\|\nabla U_{f(t)}-\nabla U_{T^{a} S_{\lambda} f_{0}}\right\|_{2}^{2}<\epsilon, t \geq 0 .
$$


The only difference to the proof of Theorem 4 is that one now has to take into account not only the spatial shifts, but also the scaling transformations which arise in Theorem 5, and this is straightforward. The condition $f(0) \in$ $\mathcal{F}_{M}$ can also be relaxed by a scaling transformation as in [8, Thm. 4].

Note added in proof. The stability of isotropic steady states is also addressed in

Wan, Y.-H.: Nonlinear stability of spherical systems in galactic dynamics. Preprint, 2000

\section{References}

[1] Aly, J. J.: On the lowest energy state of a collisionless selfgravitating system under phase space volume constraints. Monthly Notices Royal Astronomical Soc. 241, 15-27 (1989)

[2] Batt, J., Faltenbacher, W., \& Horst, E.: Stationary spherically symmetric models in stellar dynamics. Arch. Rational Mech. Anal. 93, 159-183 (1986)

[3] Batt, J., Morrison, P., Rein, G.: Linear stability of stationary solutions of the Vlasov-Poisson system in three dimensions. Arch. Rational Mech. Anal. 130, 163-182 (1995)

[4] Binney, J. \& Tremaine, S.: Galactic Dynamics. Princeton: Princeton University Press 1987

[5] Braasch, P., Rein, G., Vukadinović, J.: Nonlinear stability of stationary plasmas - an extension of the energy-Casimir method. SIAM J. Applied Math. 59, 831-844 (1999)

[6] Fridman, A. M., Polyachenko, V. L.: Physics of Gravitating Systems I, Springer-Verlag, New York 1984

[7] Guo, Y.: Variational method in polytropic galaxies. Arch. Rational Mech. Anal., 150, 209-224 (1999)

[8] Guo, Y.: On the generalized Antonov's stability criterion. Contem. Math. 263, 85-107 (2000) 
[9] Guo, Y., Rein, G.: Stable steady states in stellar dynamics. Arch. Rational Mech. Anal. 147, 225-243 (1999)

[10] Guo, Y., Rein, G.: Existence and stability of Camm type steady states in galactic dynamics. Indiana University Math. J., 48, 1237-1255 (1999)

[11] Guo, Y., Strauss, W.: Nonlinear instability of double-humped equilibria. Ann. Inst. Henri Poincaré 12, 339-352 (1995)

[12] Guo, Y., Strauss, W.: Instability of periodic BGK equilibria. Comm. Pure Appl. Math. 48, 861-894 (1995)

[13] Lieb, E. H., Loss, M.: Analysis. American Mathematical Society, Providence 1996

[14] Lions, P.-L.: The concentration-compactness principle in the calculus of variations. The locally compact case. Part 1. Ann. Inst. H. Poincaré 1, 109-145 (1984)

[15] Pfaffelmoser, K.: Global classical solutions of the Vlasov-Poisson system in three dimensions for general initial data. J. Diff. Eqns. 95, 281-303 (1992)

[16] Rein, G.: Nonlinear stability for the Vlasov-Poisson system-the energy-Casimir method. Math. Meth. in the Appl. Sci. 17, 1129-1140 (1994)

[17] ReIN, G.: Flat steady states in stellar dynamics - existence and stability. Commun. Math. Phys. 205, 229-247 (1999)

[18] ReIN, G.: Stability of spherically symmetric steady states in galactic dynamics against general perturbations. Arch. Rational Mech. Anal. 161, 27-42 (2002)

[19] Rein, G., Rendall, A. D.: Compact support of spherically symmetric equilibria in non-relativistic and relativistic galactic dynamics. Math. Proc. Camb. Phil. Soc. 128, 363-380 (2000) 
[20] Schaeffer, J.: Global existence of smooth solutions to the VlasovPoisson system in three dimensions. Commun. Part. Diff. Eqns. 16, 1313-1335 (1991)

[21] Wan, Y.-H.: On nonlinear stability of isotropic models in stellar dynamics. Arch. Rational Mech. Anal. 147, 245-268 (1999)

[22] Wolansky, G.: On nonlinear stability of polytropic galaxies. Ann. Inst. Henri Poincaré, 16, 15-48 (1999) 